POS $\quad$ PROCEEDINGS

\title{
Relativistic Jets from black hole accretion disc
}

\section{Indranil Chattopadhyay*}

ARIES, Manora Peak, Nainital 263002, India

E-mail: indra@aries.res.in

\section{Mukesh K. Vyas}

ARIES, Manora Peak, Nainital 263002, India

E-mail: mukeshkvys@gmail. com

\section{Kuldeep Singh}

ARIES, Manora Peak, Nainital 263002, India

E-mail: kuldeep@aries.res.in

\begin{abstract}
Astrophysical jets from AGNs and microquasars are often relativistic and collimated. We study magnetic and radiative driving of jets to address these issues. The plasma is described by a relativistic equation of state which depends on the composition. We show that the matter content may not affect the streamline of magnetically driven jets, but the poloidal velocity and temperature distribution strongly depend on the composition of the jet. We also discuss the salient features of radiatively driven jets. Although consensus in the community precludes radiation driving to be an effective acceleration mechanism, we show that it is certainly not the case. For black holes surrounded by luminous discs, jets may be accelerated up to Lorentz factors of $\sim$ a few for baryon dominated jets. Interestingly, the terminal Lorentz factor may reach to a value of a few tens for lepton dominated jets. We also show that internal shocks driven by radiation are also possible in jets. Moreover, a temperature dependent scattering cross-section can produce relativistic jets that are launched with very low speeds and quite moderate temperatures, conditions which are expected in the inner region of the accretion discs. Although we have studied magnetic driving and radiative driving separately, it is apparent that both processes should be incorporated in order to solve the collimation and acceleration enigma of astrophysical jets.
\end{abstract}

High Energy Phenomena in Relativistic Outflows VII - HEPRO VII

9-12 July 2019

Facultat de Fisica, Universitat de Barcelona, Spain

${ }^{*}$ Speaker. 


\section{Introduction}

Astrophysical jets are ubiquitous and have been associated with active galactic nuclei (AGN), microquasars or X-ray binaries, GRBs and even YSOs. However, only jets around compact objects are relativistic. Since black holes do not have any environment, an outward, collimated flow has to originate from the accreting matter itself. However, there is little consensus on the possible mechanism to launch the jet. One of the most popular process to launch a jet is the so-called Blandford-Znajek (BZ) mechanism [1], however, the jet launched by this process is essentially Poynting flux dominated, while there are many studies which give indirect evidence that the large scale jet is matter dominated [2]. Moreover, there are few observations which contradicts the jet power as estimated considering only the BZ mechanism [3]. Furthermore, the jet spectral states correlate with the accretion states in microquasars $[4,5,6]$, which actually suggest that the jets are launched from the accretion disc. There are other jet launching mechanisms proposed in the literature like the Blandford Payne mechanism [7] or accretion shock generated bipolar outflows [8]. Observations also show that jets are launched from a region very close to the horizon [9]. Since large scale jets are matter dominated, they correlate with different states of the accretion disc in microquasars, and originate very close to the $\mathrm{BH}$, we consider matter dominated jets. In this paper we do not study the jet launching mechanisms, but we study jet acceleration processes.

General accretion discs (not Keplerian disc as studied by [10]) are sources of intense high energy radiation. And such high energy radiation directly 'stare' at the narrow jet as it moves away from the accretion disc, and therefore may deposit enough energy and momentum on the jet to accelerate it to very high speeds. However, it has been shown that an optically thin plasma plying through a radiation field, may suffer from radiation drag effect. Such a radiation drag effect is proportional to the velocity and hence its effect becomes important for relativistically moving jets $[11,12]$. However, for a disc where most of the radiation is coming from the inner part of the disc, it has been shown that the effect of radiation drag is quite limited [13, 14, 15, 16]. We have studied jets in the general relativistic and Compton scattering regime.

Jet acceleration by radiation pressure is a mechanism which has been studied in detail $[13,14$, $15,16,19,18]$. Nonetheless, there are many observations of relativistic jets from low luminosity objects. In those cases magnetic acceleration is the only known process to produce relativistic jets. In both radiatively and magnetically driven regimes, we use relativistic equation of state, as proposed by [17].

\section{Radiatively Driven Jets}

A jet cannot be relativistic only by thermal driving. If we consider a purely ideal flow, then at the base of the jet the three-velocity should be zero. The Bernoulli parameter which in Schwarzschild metric is $E=h \gamma(1-2 / r)$ [20], where $h$ is the specific enthalpy, $\gamma$ the total Lorentz factor and $r$ the radial coordinate. At the jet base, it becomes $E=h_{\mathrm{b}}\left(1-2 / r_{\mathrm{b}}\right)$, as $\gamma_{\mathrm{b}} \approx 1$. Here, suffix 'b' signifies quantities at the jet base. At infinite distance $h_{\infty} \rightarrow 1$, and the terminal speed is given by

$$
v_{\infty}=\left(1-\frac{1}{E^{2}}\right)^{1 / 2}
$$


To achieve a Lorentz factor of 10 one would require temperatures at the jet base to be around $T_{\mathrm{b}} \sim 10^{13} \mathrm{~K}$. Such high temperatures are not likely to exist in accretion discs. So we need some mechanism to take the outflowing matter from base velocities of $v_{\mathrm{b}} \sim 0$ to very high values.

The equations of motion of radiatively driven jets in steady state are:

$$
u^{r} \frac{d u^{r}}{d r}+\frac{1}{r^{2}}=-\left(1-\frac{2}{r}+u^{r} u^{r}\right) \frac{1}{e+p} \frac{d p}{d r}+\rho_{e} \frac{\sqrt{g^{r r}} \gamma^{3}}{(e+p)} \mathscr{I}^{r}
$$

Here, $u^{r}$ is the radial four velocity, $\gamma$ is the bulk Lorentz factor, $\rho_{e}, e$ and $p$ are the lepton mass density, energy density and the isotropic pressure of the fluid, respectively. If $u^{t}$ is the contravariant time component of the four velocity then the Lorentz factor is $\gamma=\left(-u_{t} u^{t}\right)^{1 / 2}=\left(1-v^{2}\right)^{-1 / 2}$ of the jet, where $v=\sqrt{\left(-u_{r} u^{r} / u_{t} u^{t}\right)}$ is the three velocity in the radial direction. It means $u^{r}=$ $\sqrt{g^{r r}} \gamma \nu$, where $g^{r r}$ is the $r r$ component of the metric tensor. Further, the radiative contribution to the momentum in equation (2.2) can be expressed as

$$
\mathscr{I}^{r}=\frac{\sigma}{m_{e}}\left[\left(1+v^{2}\right) \mathscr{R}_{1}-v\left(g^{r r} \mathscr{R}_{0}+\frac{\mathscr{R}_{2}}{g^{r r}}\right)\right]
$$

where the first three moments of the specific intensity of the radiation field are $\mathscr{R}_{0}, \mathscr{R}_{1}$ and $\mathscr{R}_{2}$ and are identified as the radiation energy density, the flux and the pressure, respectively. The conservation of the number density flux can be integrated and transformed into the mass outflow rate equation:

$$
\dot{M}_{o}=\rho u^{r} \mathscr{A}
$$

where, $\mathscr{A}$ is the cross-sectional area of the jet. The first law of thermodynamics is given by,

$$
\frac{d e}{d r}-\frac{e+p}{\rho} \frac{d \rho}{d r}=-\frac{\gamma \rho_{e}\left(1-\chi_{c}\right) R_{t}}{\sqrt{g^{r r}}}
$$

where,

$$
\sigma=\chi_{c} \sigma_{T}=\left[\frac{1}{1+\left(\frac{T_{e}}{4.5 \times 10^{8}}\right)^{0.86}}\right] \sigma_{T}
$$

and

$$
R_{t}=\frac{\sigma_{T}}{m_{e}}\left[\frac{g^{r r} \mathscr{R}_{0}}{v}+\frac{v \mathscr{R}_{2}}{g^{r r}}-2 \mathscr{R}_{1}\right]
$$

Equations (2.2-2.5) can be solved once the equation of state (EoS) is supplied and in physical dimensions it is given by:

$$
e=\rho_{e} c^{2} f
$$

where the dimensionless $f$ is

$$
f=(2-\xi)\left[1+\Theta\left(\frac{9 \Theta+3}{3 \Theta+2}\right)\right]+\xi\left[\frac{1}{\eta}+\Theta\left(\frac{9 \Theta+3 / \eta}{3 \Theta+2 / \eta}\right)\right] .
$$

Here, $\Theta=k T /\left(m_{e} c^{2}\right)$ is the dimensionless temperature $(T)$ and $k$ is the Boltzmann constant, $\xi(=$ $\left.n_{p^{+}} / n_{e^{-}}\right)$is the ratio of the number densities of protons and electrons, and $\eta\left(=m_{e} / m_{p^{+}}\right)$is the 
mass ratio between electrons and protons. This EoS is abbreviated as CR EoS. The expressions of $\Gamma, N, a$ and $h$ (in geometric units) are obtained through

$$
N=\frac{1}{2} \frac{d f}{d \Theta} ; \Gamma=1+\frac{1}{N} ; a^{2}=\frac{\Gamma p}{e+p}=\frac{2 \Gamma \Theta}{f+2 \Theta} ; h=\frac{f+2 \Theta}{\tau}
$$

Here $\tau$ is a function of of the jet composition and is defined as $\tau=2-\xi+\xi / \eta$.

The methodology to solve equations (2.2-2.5) has been laid down in a series of papers [19, 20, 18, 21]. Assuming the Thomson scattering cross-section i. e., when $\chi_{c}=1$, we showed that relativistic terminal speeds could be achieved but the temperature of the jet at the base was found to be very high $[19,18]$. Consideration of Compton scattering cross-section seems therefore counter-intuitive to achieve relativistic jets. The Compton scattering cross-section is smaller than the Thompson scattering cross-section, and therefore the momentum transferred would be accordingly reduced. However, in the Compton regime radiation energy could be transferred to the jet. The energy transferred to the jet increases the temperature and therefore more than compensates for the reduction in the cross-section, to the extent that it can even drive a bound flow as a transonic outflowing jets

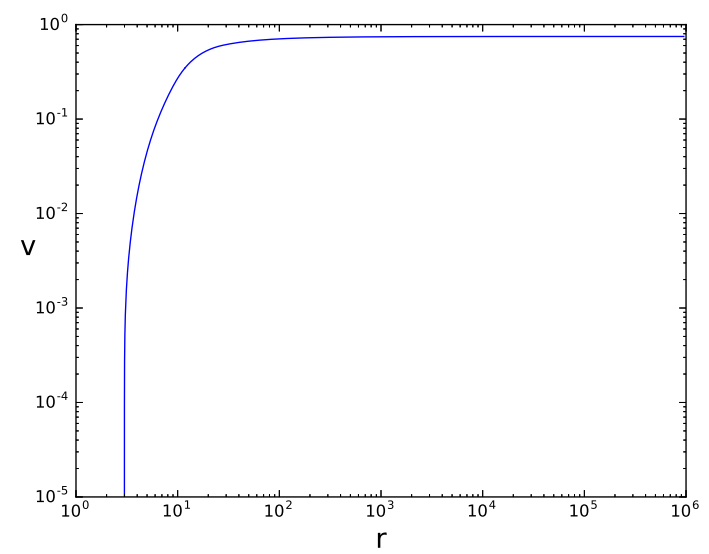

Figure 1: The velocity distribution of a jet which is driven by a disc with luminosity $\ell=2$ (in units of Eddington luminosity), and a relativistic Bernoulli parameter $E=0.57$.

In Figures 1 and 2 we show that for intense enough radiation fields, even bound matter $(E<1)$ can also be driven as relativistic outflows. Very interestingly the jet at the base starts with negligible velocity and non-relativistic temperature, and therefore matches the inner boundary condition (i.e. low jet base values of $v$ and $T$ ) for jets launched from accretion disc.

\section{Magnetically Driven Jets}

The first consistent magnetized wind solution was obtained by Weber \& Davis [22]. They showed that the physically meaningful magnetically driven wind passes through the slow, the Alfven and the fast critical points. A magnetized wind model which was inspired by the WeberDavis model, used a variable adiabatic index equation of state and showed the dependence of magnetized wind solutions on the composition of the flow [23]. Presently, we follow the papers by Vlahakis \& Konigl and Polko et. al. [24, 25] and use the method of radial self similarity to obtain 


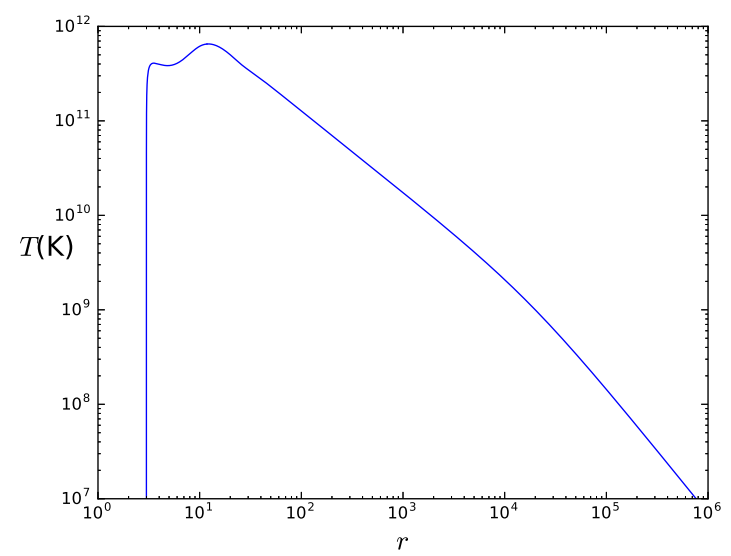

Figure 2: The temperature distribution of a jet which is driven by a disc with luminosity $\ell=2$ (in units of Eddington luminosity), and a relativistic Bernoulli parameter $E=0.57$.

the streamlines and solve the relativistic magneto-hydrodynamic equations. However, unlike these authors, we used the relativistic CR EoS i.e, equations 2.8,2.9 to describe the thermodynamics of the flow. The equations of motion are:

The mass conservation equation:

$$
\frac{\partial(\gamma \rho)}{\partial t}+\nabla \cdot(\mathbf{v} \gamma \rho)=0
$$

The momentum conservation equation

$$
\gamma \rho\left(\frac{\partial}{\partial t}+\mathbf{v} \cdot \nabla\right)(h \gamma \mathbf{v})=-\nabla p+\frac{J^{0} \mathbf{E}+\mathbf{J} \times \mathbf{B}}{c} .
$$

The first-law of thermodynamics

$$
\left(\frac{\partial}{\partial t}+\mathbf{v} \cdot \nabla\right) e+p\left(\frac{\partial}{\partial t}+\mathbf{v} \cdot \nabla\right)\left(\frac{1}{\rho}\right)=0 .
$$

In addition, the Maxwell's equations, the ideal MHD condition and the gas EoS (equation 2.8) were used to solve the above equations of motion. We showed that the solutions depend on a large number of parameters [26].

In Fig. 3a we plot the two jet streamlines which are the same in all the respects but differs only by the 'current distribution' parameter $F$ (see equation 24a of [24]). For $F=0.81$, the flow passes through the Alfven and the fast points (solid, black), while for $F=0.85$ the solution passes only through Alfven point (dashed, red). The solution that passes through both the critical points are over-collimated and possibly the flow terminates in a shock on the axis of rotation. The jet that passes only through one critical point does not terminate, but is slower (poloidal velocity $v_{\mathrm{p}}$ ). The Alfven Mach number $M$ shows minor differences. Both jets flip the azimuthal velocity $\left(v_{\phi}\right)$. This is because the magnetic field transfers an energy $E_{S}$ to the matter $E_{M}$. The magnitude of the poloidal and azimuthal magnetic field components $\left(B_{\mathrm{p}}\right.$ and $\left.B_{\phi}\right)$ decrease as the jet moves outward. The Lorentz factor of the jet through both the critical points is ultra relativistic, $\sim 100$. In this case the temperature $T$ and the adiabatic index $\Gamma$ are similar for both the jets. In Singh \& Chattopadhyay $2019 b$ [26] we showed that it is very important to consider the relativistic CR EoS. 

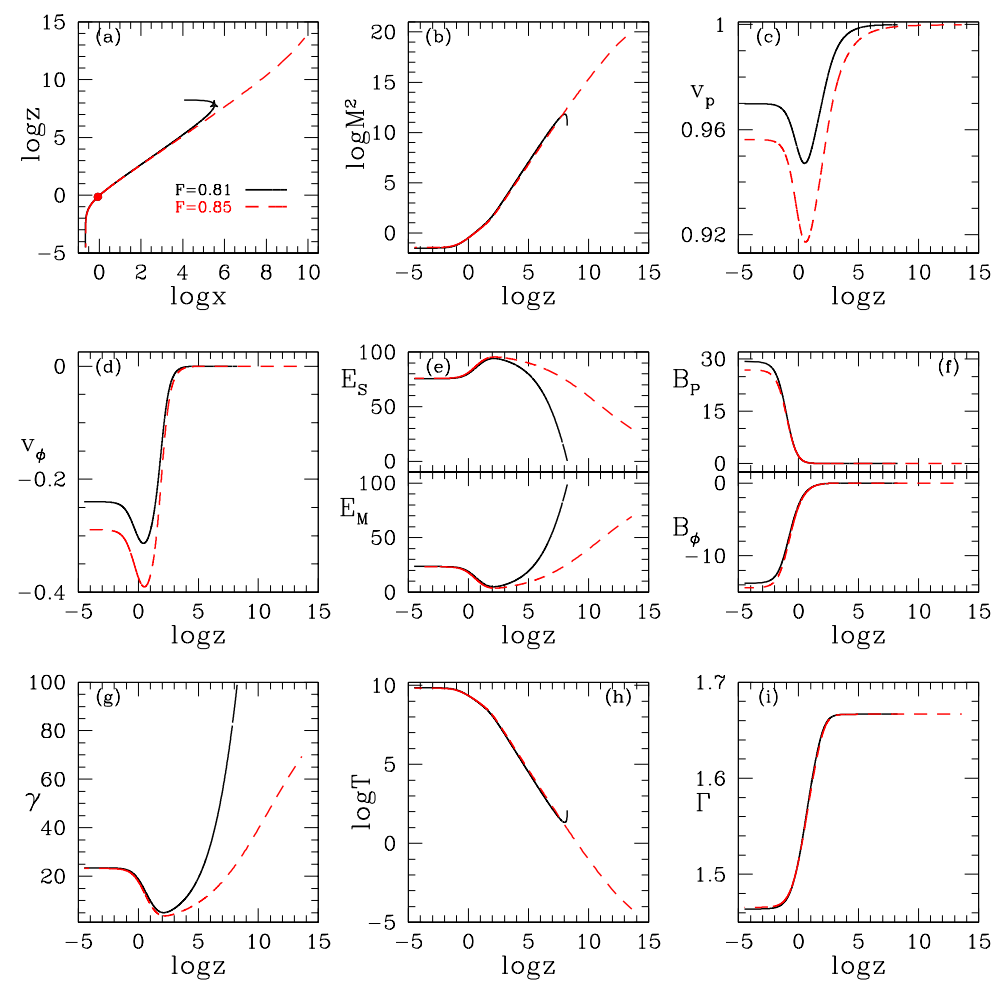

Figure 3: Flow variables like (a) streamline, (b) square of Alfven Mach number $M^{2}$, (c) poloidal velocity $v_{p}$, (d) azimuthal velocity $v_{\phi}$, (e) matter and electromagnetic components of the Bernoulli parameter $E_{M}$ and $E_{S}$, respectively, (f) poloidal and azimuthal components of the magnetic field $B_{p}$ and $B_{\phi}$, (g) Lorentz factor $\gamma$, (h) $\log T$ and (i) adiabatic index $\Gamma$, for jets described by $F=0.81$ (solid, black) which passes through the Alfven (red dot) and the fast point (black triangle), while for $F=0.85$ (dashed, red) the jet passes only through the Alfven point. The common jet parameters are Bernoulli parameter $E=99.1507, \theta_{A}=50, \psi_{A}=55$, $q=500$, and square of Alfven radius $x_{A}^{2}=0.75$ (in units of light cylinder). The jet is composed of electrons and protons. See Singh \& Chattopadhyay 2019b [26], for more details.

\section{Conclusion}

We showed that radiative pressure can produce relativistic jets with proper base temperatures and velocities, but the limitation is that the underlying accretion disc has to be highly luminous. We showed that magnetic driving can produce ultra relativistic jets. In a previous paper we also showed that using the CR EoS produces a proper temperature profile. The limitation of this work is that we have not extended it to general relativity and therefore the slow critical point is not formed. The absence of such a slow point prevents us to connect the magnetic jet solution to the underlying accretion disc parameters.

\section{Acknowledgment}

IC acknowledges ARIES and CSIR to provide the travel fund to attend the conference. IC also acknowledges the LOC of HEPROVII for local hospitality. 


\section{References}

[1] Blandford R. D., Znajek R. L., 1977, MNRAS, 179, 433

[2] Reynolds C. S., Fabian A. C., Celotti A., Rees M. J., 1996, MNRAS, 283, 873

[3] Fender R. P., Gallo E., Russell D., 2010, MNRAS, 406, 1425.

[4] Gallo E., Fender R. P., Pooley G. G., 2003, MNRAS, 344, 60

[5] Fender R. P., Belloni T. M., Gallo E., 2004, MNRAS, 355, 1105

[6] Fender R. P., Gallo E., 2014, Space Sci. Rev., 183, 323

[7] Blandford R. D., Payne D. G., 1982, MNRAS, 199, 883

[8] Chakrabarti S. K., 1999, A\&A, 351, 185

[9] Junor W., Biretta J. A., Livio M., 1999, Nat, 401, 891

[10] Shakura N. I., Sunyaev R. A., 1973, A\&A, 24, 337

[11] Icke V., 1989, A\&A, 216, 294

[12] Fukue J., 1996, PASJ, 48, 631

[13] Chattopadhyay I., Chakrabarti S. K., 2000, Int. Journ. Mod. Phys. D, 9, 717

[14] Chattopadhyay I., Chakrabarti S. K., 2002, MNRAS, 333, 454

[15] Chattopadhyay I., Das S., Chakrabarti S. K., 2004, MNRAS, 348, 846

[16] Chattopadhyay I., 2005, MNRAS, 356, 145

[17] Chattopadhyay I., Ryu D., 2009, ApJ, 694, 492

[18] Vyas M. K., Chattopadhyay I., 2018, A\&A, 614, A51

[19] Vyas M. K., Kumar R., Mandal S., Chattopadhyay I., MNRAS, 453, 2992

[20] Vyas M. K., Chattopadhyay I., 2017, MNRAS, 369, 3270

[21] Vyas M. K., Chattopadhyay I., 2019, MNRAS, 482, 4230

[22] Weber E. J., Davis L. J., 1967, ApJ, 148, 217

[23] Singh K., Chattopadhyay I., 2019a, MNRAS, 486, 3506

[24] Vlahakis N., Konigl A., 2003, ApJ, 596, 1080

[25] Polko P., Meier D. L., Markoff S. 2010, APJ, 723, 1343

[26] Singh K., Chattopadhyay I., 2019b, MNRAS, 488, 5713 